\title{
Exacerbation of Congenital Hydronephrosis as the First Presentation of COVID-19 Infection in Children: A Case Series
}

\section{Masoumeh Mohkam}

Shahid Beheshti University of Medical Sciences

Mahnaz Jamee ( $\square$ jamee@sbmu.ac.ir)

Shahid Beheshti University of Medical Sciences https://orcid.org/0000-0002-0732-9170

Farshid Kompani

Golestan University of Medical Sciences and Health Services

Mitra Khalili

Shahid Beheshti University of Medical Sciences

\section{Atena Seifi}

Shahid Beheshti University of Medical Sciences

\section{Leily Mohajerzadeh}

Shahid Beheshti University of Medical Sciences

\section{Research Article}

Keywords: Hydronephrosis, UPJO, Kidney, COVID-19, Child

Posted Date: October 18th, 2021

DOI: https://doi.org/10.21203/rs.3.rs-920932/v1

License: (1) This work is licensed under a Creative Commons Attribution 4.0 International License. Read Full License 


\section{Abstract}

\section{Background:}

Congenital hydronephrosis is one of the most common abnormalities of the upper urinary tract, which can be exacerbated by a variety of intrinsic or extrinsic triggers. The urinary tract system is one of the major organs complicated by COVID-19 infection.

\section{Case presentations:}

Here we report five patients with an established diagnosis of congenital hydronephrosis, who presented with acute abdominal pain and fever and an abrupt increase in the anteroposterior pelvic diameter (APD). Patients had a previous stable course and were under regular follow-up with serial ultra-sonographic studies. They underwent surgery or supportive treatment due to the later exacerbation of hydronephrosis. Based on the clinical and imaging findings, no plausible etiologies for these exacerbation episodes, including infection, nephrolithiasis or abdominal masses, could be postulated. The common aspect in all these patients was the evidence of a COVID-19 infection.

\section{Conclusions:}

Infection with COVID-19 in children with antenatal hydronephrosis may exacerbate the degree of hydronephrosis and renal APD in ultrasonography, which itself may be mediated by the increase in inflammatory mediators.

\section{Introduction}

Congenital hydronephrosis is one of the most common abnormalities of the upper urinary tract diagnosed by prenatal ultrasonography. Hydronephrosis, as defined by a renal pelvic diameter of more than $5 \mathrm{~mm}$ according to the Bristol group, is found in approximately $1-5 \%$ of all pregnancies [1]. Now it has become clear that $70-98 \%$ of the mild isolated congenital hydronephrosis cases improve spontaneously during follow-up without any need for surgical intervention [2].

More than forty percent of congenital hydronephrosis cases are due to transient etiologies [3]; physiologic etiologies comprise $15 \%$, ureteropelvic junction obstruction (UPJO) $11 \%$, and vesicoureteral reflex $9 \%$ of etiologies, while other etiologies are encountered less frequently [4]. Based on the anteroposterior pelvic diameter (APD) grading system (classified as grade 1 to 4 over a scale of $5 \mathrm{~mm}$ ), hydronephrosis grades 1 to 4 are found to resolve in $80.0 \%, 41.2 \%, 13.1 \%$, and $2.5 \%$ of patients over a 4 -year-period, respectively [5].

The most common types of post-natal hydronephrosis are the transient and physiologic ones in which a close follow-up with serial ultra-sonographic studies is required if the first renal function tests are normal and no unitary tract infection (UTI) or hypertension is present. 
Antibiotic prophylaxis is recommended especially in moderate to severe cases of hydronephrosis [4], and may be associated with an improvement rate of $47 \%$ in a 12-month follow-up [6].

An acute increase in APD sometimes occurs with accompanying complications in mild to moderate cases of hydronephrosis following infections, urolithiasis, blood clots, trauma, abdominal masses, fibrosis, retroperitoneal lymphadenopathy, and malignancies.

In the present era of widespread COVID-19 infection, a heterogeneous spectrum of manifestations is plausible in the setting of COVID-19. The renal manifestations of COVID-19 in the acute phase usually range from mild proteinuria, leukocyturia, or asymptomatic hematuria to severe acute kidney injury and blood pressure changes $[7,8]$.

Herein, we aim to report presentation of severe hydronephrosis in children with a previous stable mild post-natal hydronephrosis, complicated by COVID-19 infection.

\section{li. Cases Reports}

\section{Case 1:}

A 6-year-old male was referred to the emergency department of Mofid children's Hospital with complaints of abdominal pain and vomiting in the 2020 late spring.

The case was under follow-up due to congenital hydronephrosis in our nephrology clinic. On neonatal ultrasonography, the APD of the renal pelvis was $13 \mathrm{~mm}$ on the right and $12 \mathrm{~mm}$ on the left. The blood pressure, renal function tests, urinalysis, and voiding cystourethrography (VCUG) were normal. The APD had gradually decreased to $6 \mathrm{~mm}$ on the right and $5 \mathrm{~mm}$ on the left at 5 years of age ( 6 months before the last admission) in our serial follow-ups together with a normal bladder appearance and post-voiding residual volume.

On admission, the weight was $22 \mathrm{~kg}$ (65\% percentile) and blood pressure was 100/60 mmHg.. On physical examination, there was mild generalized abdominal and right costovertebral angle tenderness and there was no fever or evidence of a respiratory or another gastrointestinal problem.

The hemoglobin level was $12.8 \mathrm{~g} / \mathrm{dl}$ and C-reactive protein (CRP) was in the normal range and urinalysis showed 3-4 RBCs with no WBC or protein.

Abdominal ultrasonography was performed on the first day of admission which showed the size of the kidneys to be $108 \mathrm{~mm}$ on the right and $80 \mathrm{~mm}$ on the left and a severe hydronephrosis on the right with APD of $35 \mathrm{~mm}$. The APD of the left kidney and the echogenicity of both kidneys were normal. The ultrasonography of the urinary tract was repeated after 2 days and on the last imaging, the APD increased to $45 \mathrm{~mm}$ while clinically abdominal pain persisted without any localized tenderness during this time. 
Spiral abdominopelvic computerized tomography (CT) scan showed the same findings on ultrasonography without detection of urolithiasis. TC-99m Diethylenetriamine pentaacetate (DTPA) scan showed obstructive hydronephrosis on the right kidney.

The patient underwent surgery due to symptomatic obstruction and the findings during surgery were moderate obstruction and marked inflammation at the ureteropelvic junction (UPJ) without complete obstruction. He became febrile the day following the surgery and the evaluations showed a positive CRP and also a positive polymerase chain reaction (PCR) test for the COVID-19 virus.

The chest CT scan showed the characteristic pulmonary involvement in favor of COVID-19 infection. The patient was transferred to the COVID-19 ward and was discharged after one week with a good general condition.

On the day of discharge, the ultrasonography showed mild hydronephrosis with an RPD of $8 \mathrm{~mm}$ and also normal laboratory tests and blood pressure.

The patient was followed quarterly for one year and showed no abnormal clinical or laboratory findings.

\section{Case 2:}

A 13-month-old female was referred to the outpatient clinic of our hospital with complaints of mild fever and abdominal pain in February 2020. Urinalysis and urine culture were done due to a prior history of prenatal mild hydronephrosis with stable clinical course. Urine analysis showed pyuria and oral cefixime was prescribed with a presumed urinary tract infection diagnosis. The patient became afebrile within two days and began to develop diarrhea and vomiting. Further evaluations showed a positive PCR test for COVID-19. The patient underwent abdominal ultrasonography which reported a severe degree of hydronephrosis.

The patient underwent pyeloplasty due to the uptake defect on DMSA (Dimercaptosuccinic acid) scan, signs of obstruction in DTPA scan, and exacerbation of hydronephrosis after the recovery period of COVID-infection.

\section{Case 3:}

A 3-year-old female was followed up due to a history of mild hydronephrosis and anti-reflux surgery at one year of age. She had no history of UTI and was quite stable clinically with only mild unilateral hydronephrosis on renal ultrasonography two months before referral in July 2020.

She was referred due to fever for a few days and was admitted with a presumed diagnosis of UTI due to pyuria and proteinuria in urinalysis. On repeat ultrasonography, severe hydronephrosis was detected in the ipsilateral kidney and on the DMSA scan a severe uptake defect of the hydronephrotic kidney was detected. In recent history the child had exposure to a case of COVID-19 one week before admission.

\section{Case 4:}


The patient was a 6-year-old female who was under follow-up due to prenatal hydronephrosis and one episode of UTI. There was no evidence of vesicoureteral reflux in her infantile evaluation.

Pyeloplasty was done at one year of age due to the obstructive pattern on the DTPA scan. At her postsurgical follow-up, the APD in renal ultrasonography was normal and the clinical course was stable.

Three months after the last visit (in November 2020) she was referred to our outpatient clinic due to occasional complaints of abdominal pain and anorexia. In the first evaluation urinalysis was normal and on ultrasonography, a severe degree of hydronephrosis was reported. The past medical history revealed infection with COVID-19 a week before referral.

DTPA scan showed a non-obstructive pattern despite the severe hydronephrosis.

The patient is now under follow-up with decreasing APD in serial ultrasonography and normal clinical and laboratory data.

\section{Case 5:}

The case was a 5-year-old male who was under follow-up due to bilateral hydronephrosis from birth. There was no history of UTI and no evidence of vesicoureteral reflux in the neonatal evaluation. Her evaluation in infancy showed a non-obstructive pattern on DTPA scan and serial follow-up revealed improving course bilaterally.

Six months before the last visit, renal function tests, urinalysis, and blood pressure were normal and the renal ultrasonography showed an improving trend of hydronephrosis.

On the last visit in May 2021, kidney ultrasonography showed a marked bilateral increase in APD despite stable clinical course and the history revealed infection with COVID-19 virus one month before referral. A DTPA scan was ordered and is being awaited.

\section{lii. Discussion}

Ureteropelvic junction obstruction (UPJO), classified as congenital anomalies of the kidney and urinary tract (CAKUT) with an obstructive pattern, constitutes the most important etiology of hydronephrosis and also one of the common causes of chronic kidney disease (CKD) in children. Severe UPJO is associated with obstruction to urine flow from the renal pelvis to the ureter and can result in ultimately complete loss of the involved kidney [9].

In childhood, UPJO is usually congenital and can be diagnosed by the prenatal kidney and urinary tract ultrasonography (usually in the 3rd trimester). The acquired cases of UPJO are divided into intrinsic and extrinsic etiologies. The extrinsic causes are associated with outside pressure to the ureteropelvic junction or proximal ureters elicited by fibrosis, mass, or retroperitoneal lymphadenopathy. 
The intrinsic causes of UPJO result from urolithiasis, radiation, or chronic inflammation, which cause scars in the ureteral wall or para-ureteral region. According to some studies, one of the important etiologies for exacerbation of UPJO is urolithiasis, especially in the COVID-19 pandemic. In a recent study in Turkey on 149 patients with ureteral stones, it was shown that the number of emergency cases with admission during the COVID-19 epidemic increased by 3-fold in comparison with the time before the epidemic. This finding together with the simultaneous higher incidence of severe hydronephrosis in these patients can be the result of delay in referral to the clinic as a result of personal isolation. This delayed referral can also explain some cases with a higher creatinine level [10].

The inflammatory mechanisms can be a predisposing factor for UPJO among the different abovementioned etiologies. Moreover, UPJO itself can trigger the production of vasoactive peptides and cytokines like interleukin- 5 and eotaxin- 2 which recruit leukocytes and form inflammatory cell infiltrate [11].

Several studies have been conducted to evaluate the relationship between inflammatory mechanisms and UPJO over the last decades. In a study by Chiou et al in 2005, the pathology of 24 ureteropelvic junction segments of 24 patients was evaluated. They noted an increased expression of IL-5, and eotaxin, and increased urothelial mast cell degranulation. The study showed increased levels of IL-5, $\gamma$-interferon, and eotaxin in the kidneys with an obstructed system, among which IL-5 and $\mathrm{y}$-interferon were highly ( $\mathrm{p}<$ 0.05) associated with the severity of obstructive uropathy [12]. In an animal-model study by Chen et al, it was shown that the establishment of unilateral ureteral obstruction is associated with increased expression of IL-33 in vimentin-positive cells of medullary layers, cortex, and UPJ stroma. They proposed that the IL-33/ST2 axis may mediate the activation of innate immune responses and results in urothelial hyperplasia [13].

Infection with SARS-COV-2 may result in persistent endothelial inflammation in different organs like the lung, heart, and kidneys [14]. According to different studies, there is a marked increase in proinflammatory cytokines like ILIB, IL-6, IL-12, IFN- $y$, IP-10, and MCP-1 in the serum of infected patients [15].

We think this marked and generalized increase in the inflammatory state may predispose patients to the exacerbation of UPJO as was observed in our patients with prior histories of COVID-19 infection.

\section{Iv. Conclusion}

Infection with SARS-COV-2 in children with antenatal hydronephrosis may exacerbate the degree of hydronephrosis and renal APD in ultrasonography which itself may be mediated by the increase in inflammatory mediators.

\section{List Of Abbreviations}

APD; anteroposterior pelvic diameter 
CAKUT; congenital anomalies of the kidney and urinary tract

CKD; chronic kidney disease

CRP; C-reactive protein

CT; computerized tomography

DMSA; dimercaptosuccinic acid

DTPA; Diethylenetriamine pentaacetate

IL; interleukin

PCR; polymerase chain reaction

UPJO; ureteropelvic junction obstruction

UTI; unitary tract infection

VCUG; voiding cystourethrography

\section{Declarations}

\section{Ethics approval and consent to participate:}

This study was approved by the Shahid Beheshti University of Medical Sciences Ethics Committee. Informed consent was obtained from the parents of the patients prior to being included in the study.

\section{Consent for publication:}

Written informed consent for publication was obtained from the parents of the patients.

\section{Availability of data and material:}

Not applicable

\section{Competing interests:}

The authors have no conflict of interest.

\section{Funding:}

The authors received no specific funding for this research.

\section{Acknowledgements:}


We thank patients and their families for their participation in this study.

\section{References}

1. Nguyen HT, Herndon CD, Cooper C, et al. The Society for Fetal Urology consensus statement on the evaluation and management of antenatal hydronephrosis. J. Pediatr. Urol. 2010 Jun;6(3):212-31.

2. Yalçınkaya F, Özçakar ZB. Management of antenatal hydronephrosis. Pediatr. Nephrol. 2020 Dec;35(12):2231-2239.

3. Kari JA, Habiballah S, Alsaedi SA, et al. Incidence and outcomes of antenatally detected congenital hydronephrosis. Ann Saudi Med. 2013 May-Jun;33(3):260-4.

4. Woodward M, Frank D. Postnatal management of antenatal hydronephrosis. BJU Int. 2002 Jan;89(2):149-56.

5. Jung J, Lee JH, Kim KS, et al. Utility of Society for Fetal Urology and Anteroposterior Pelvic Diameter Grading Systems for Estimating Time to Resolution of Isolated Hydronephrosis: A Single Center Study. J Urol. 2020 Nov;204(5):1048-1053.

6. Dudley JA, Haworth JM, McGraw ME, et al. Clinical relevance and implications of antenatal hydronephrosis. Arch Dis Child Fetal Neonatal Ed. 1997 Jan;76(1):F31-4.

7. Cheng $Y$, Luo R, Wang $K$, et al. Kidney disease is associated with in-hospital death of patients with COVID-19. Kidney Int. 2020;97(5):829-838.

8. Mohkam M, Mirzaee M, Abdollah Gorgi F, et al. Renal Involvement in COVID-19 Among Iranian Children [Research Article]. Arch Pediatr Infect Dis. 2021;9(1):e106597.

9. Bilge I. Symptomatology and Clinic of Hydronephrosis Associated With Uretero Pelvic Junction Anomalies. Front Pediatr. 2020;8:520-520.

10. Gul M, Kaynar M, Yildiz M, et al. The Increased Risk of Complicated Ureteral Stones in the Era of COVID-19 Pandemic. J Endourol. 2020 Aug;34(8):882-886.

11. Al Aaraj MS, Badreldin AM. Ureteropelvic Junction Obstruction. StatPearls. Treasure Island (FL): StatPearls Publishing Copyright @ 2021, StatPearls Publishing LLC.; 2021.

12. Chiou YY, Shieh CC, Cheng HL, et al. Intrinsic expression of Th2 cytokines in urothelium of congenital ureteropelvic junction obstruction. Kidney Int. 2005 Feb;67(2):638-46.

13. Chen WY, Yang JL, Wu YH, et al. IL-33/ST2 axis mediates hyperplasia of intrarenal urothelium in obstructive renal injury. Exp. Mol. Med. 2018 Apr 20;50(4):1-11.

14. Patel SK, Juno JA, Lee WS, et al. Plasma ACE2 activity is persistently elevated following SARS-CoV-2 infection: implications for COVID-19 pathogenesis and consequences. Eur Respir J. 2021;57(5):2003730.

15. Huang $C$, Wang $Y, L i X$, et al. Clinical features of patients infected with 2019 novel coronavirus in Wuhan, China. Lancet (London, England). 2020 Feb 15;395(10223):497-506. 